\title{
Development of a Photoplethysmogram Based Heart Abnormality Detection Technique
}

\author{
Lina Fadhilah Umadi, Siti Nurfarah Ain Mohd Azam and Khairul Azami Sidek \\ Department of Electrical and Computer Engineering, \\ International Islamic University Malaysia \\ P. O. Box 10, Jalan Gombak, 50728 Kuala Lumpur \\ E-mail:azami@iium.edu.my
}

\begin{abstract}
In this study, the development of Cardioid based graph photoplethysmogram heart abnormality detection technique is presented. PPG signals in this work were collected from an online public repository called MIMIC II Waveform Database, Version 3 Part 1 with sampling rate of $250 \mathrm{~Hz}$. Each recording has one minute of PPG signals. Distinctive features were extracted, and then the Cardioid based graph was plotted as the result of the differentiation of the signals. In addition, the different shapes of closedloop created were then observed and assessed. From the Cardioid loop, the area and standard deviation were computed to distinguish between normal and abnormal heartbeats. Based on the results, these values for abnormal heartbeat were higher than the value of normal heartbeat thus signifying the differences between two categories of heart conditions. Therefore, the results of this study suggest the capability of the proposed mechanisms to determine heart abnormality and act as an alternative to the current detection system.
\end{abstract}

Keywords: PPG signal, Cardioid, cardiac irregularities and heart abnormalities

\section{Introduction}

Heart disease has become the most leading cause of death in today's world. Every person in the world regardless of their age, gender and race may develop heart disease especially to those with high blood pressure and cholesterol, diabetes and smoking [1]. About 735000 people have heart attack in United States of America every year. A total of 525000 Americans had their first experience of heart attack while the other 210000 had recurrent heart attack [2].

There are several ways to diagnose heart abnormality. Before performing any test, doctors will ask some questions about the patient's personal and family medical background. Then, the doctor will perform several tests to diagnose the abnormality which includes chest x-ray, blood test, echocardiogram, cardiac catheterization, cardiac computerized tomography (CT) scan or cardiac magnetic imaging reasoning (MRI) [3]. However, these techniques have high power consumption, is invasive, and also costly. Nowadays, the primary method to detect heart abnormality is by analysing the PPG signal but due to drift, electromagnetic and biological interferences, the outcome may have some errors in the heart rate variability (HRV) signal.

Photoplethysmographic (PPG) signal, another type of biomedical signal, has the potential to mitigate the shortcomings of PPG signal due to electrode movement and muscular activity. The finger or earlobe is not part of any electrical circuit because the emitter and detector communicate through light rather than current flow in PPG circuit. PPG signal requires only one wire and this reduces the number of wires of at least three in electrocardiogram (ECG) to one in PPG which is very desirable in ambulatory situation [4]. 
In addition, PPG has been used in hospitals and clinics to monitor and analyse health status of patients. From the PPG signal acquired, blood pressure estimation, blood oxygen level, pulse rate and breathing rate can be monitored easily [5]. To acquire a PPG signal, a probe which contains a photodiode emitting light with wavelength around 900nm and a photo-detector is used as shown in Figure 1. The light emitting diode (LED) illuminates tissue of the fingertips and the photo-detector measures the small variations in light intensity which indicates the changes in the blood vessels volume. A decrease in light intensity interprets an increase of blood volume whereas an increase in light intensity interprets a decrease of blood volume [6].

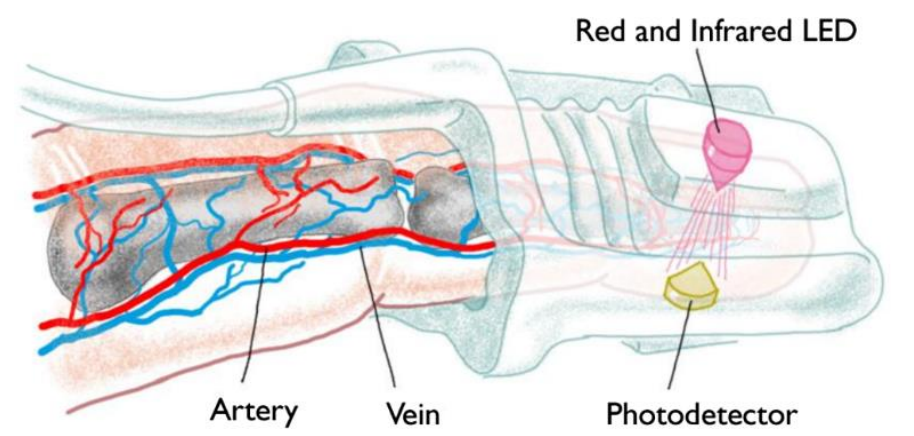

Figure 1. Acquisition of PPG Signal from Fingertip [7]

PPG pulse is divided into two phases which are anacrotic and catacrotic phases. Anacrotic phase is the rising edge of the pulse whereas catacrotic phase is the falling edge of the pulse. Anacrotic phase refers to systolic pressure when the ventricles contract and catacrotic phase refers to the diastolic pressure when the ventricles relax. A dicrotic notch depicted in Figure 2 is usually seen in catacrotic phase of normal individuals [8].

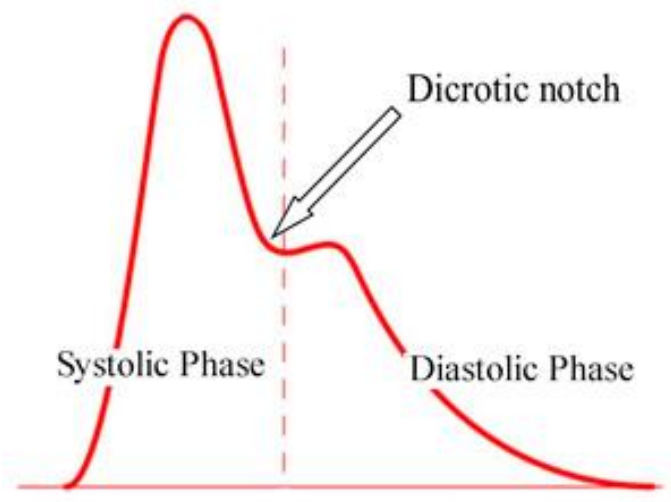

Normal arterial pulsation wave form.

Figure 2. Normal PPG Waveform [9]

The remaining sections in this paper are structured as follows; the next section will review the related works on heart abnormalities classification technique. Later, Section III, elaborates in detail on the method of the study which includes the data collection procedure, pre-processing, feature extraction and the classification mechanism. After that, in Section IV, the performance of our proposed system is discussed. Last but not least, in Section V, the study is concluded based on the experimentation and results in the previous section. 


\section{Related Works}

Saad et al. [10] proposed a detection technique of heart blocks in PPG signals by spectrum and time-frequency analysis. A total of even subjects were identified and classified as normal, first degree heart block, second degree heart block Type I, second degree heart block Type II, third degree heart block, right bundle branch block and left bundle branch block. This study interpreted cardiac abnormalities based on PPG signal which implements two types of methods which are periodogram power spectrum and spectrogram time-frequency analysis. The result shows that the subject with normal PPG signal is able to maintain higher peak frequency which is $8 \mathrm{~Hz}$ whereas subjects with heart block have a significantly low peak frequency which is below $4 \mathrm{~Hz}$.

Jose et al. [11] conducted a study on detecting cardiac arrhythmia using ballistogram (BCG) signal. In this study, 20 subjects were selected with age range of 20-40 years, considering male and female with different body mass index (BMI). The BCG signal of each subject was acquired by using a piezoelectric sensor for a period of 10 minutes. After the data was processed and heart rates of all subjects were calculated from J-J interval, the result of classification of cardiac arrhythmia can be seen from the number of heart beats per minute. Normal heart beats are around 60 to 100 times per minute. If the heart beat is less than 60 beats per minute, it is classified as bradycardia and if the heart beat is more than 100 beats per minute, it is classified as tachycardia. This technique is not much explored as the BCG device is a complicated mechanical device.

Meza [12] presented a prototype method for detection of cardiac arrhythmia from pneumatic cuff pressure course, obtained during oscillometric blood pressure measurement. In this study, pressure course samples were collected from 34 patients and used to develop an algorithm for detection of atrial fibrillation. However, based on the results, the accuracy range was only $50 \%$ for Naïve Bayes and $85.92 \%$ for Neural Network. One more disadvantage of this method is that the type of cardiac arrhythmia cannot be distinguished from one another.

Bolanos et al. [4] explored the potential use of PPG signal in determining HRV and how it relates to PPG-derived HRV in healthy individuals. This study is performed on two healthy individuals, 1 male and 1 female with ages of 24 and 25 respectively. The data was recorded for 5 minutes and 3 times per subject. In order to derive and compare different measures from both PPG signals, autoregressive (AR) modelling, Poincare' plots, cross correlation, standard deviation, arithmetic mean, skewness, kurtosis, and approximate entropy (ApEn) were used. The result shows that the correlation coefficient deviate only $1 \%$ in the first subject and 5\% in the second subject from a correlation coefficient of 1 which indicates almost perfect match between the repeated tests. The Pointcare' plots of average PPG HRV and average PPG HRV shows that the heart rate intervals cluster together in common region which indicates that PPG signal provided equivalent HRV data as derived from the PPG data.

Rubins et al. [13] performed a study which shows that PPG technique may have the potential to detect and assess arterial heart diseases associated with increasing arterial stiffness. Digital volume pulse (DVP), pulse cycle duration (T), augmentation index (AIx), reflection index (RI) and transit time of reflected wave (RTT) were evaluated in every heartbeat cycle. A total of 174 volunteers participated in this study which consists of three main groups. The first group comprises of 46 healthy subjects of age range between 23 to 35 years old, whereas the second group involves 26 healthy subjects of age range between 63 to 76 years old. On the other hand, the third group is made up of 102 patients with age range between 60 to 80 years diagnosed with cardiovascular disease. The measurements were performed in room temperature and continuously in a sitting position for one minute. The results show a clear difference between the three subject groups. For older and patients with cardiovascular disease, AIx showed higher values as compared to healthy young subjects. The values of the RTT decreased for 
higher pulse rate (or lower T) and showed lower values for patients with cardiovascular disease. Standard deviation of RTT shows greater value for patients in the third group. This study shows that all parameters are in one way or another related to each other and has the potential to be used in primary vascular diagnostic.

As a summary, biomedical signal such as PPG, BCG and PPG are capable of identifying cardiac abnormality. PPG is able to provide a low cost solution as it is easily available in the market. Thus, there is a potential for it to be used frequently in the future. Relating to this matter, based on studies from [4] and [13], we will propose a more efficient and robust PPG based heart abnormality detection technique.

\section{Methodology}

Figure 3 reviews our proposed identification scheme which comprises of the Data Collection, Pre-Processing, Feature Extraction and Classification stages. Each stage will be expounded further in the next subsections.

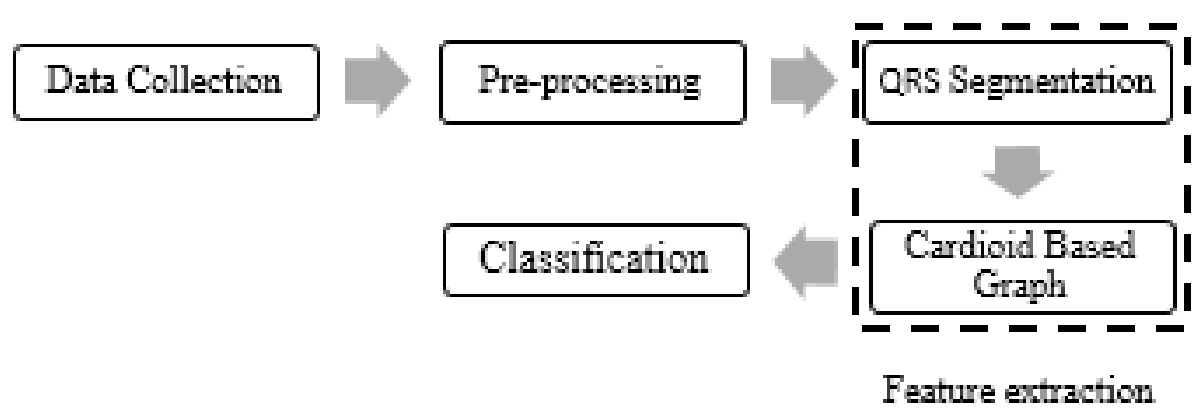

Figure 3. The Proposed Identification System

\subsection{Data Collection}

A total of 5 PPG waveforms in this work were taken from a public online databank called PhysioNet. The data were taken from MIMIC II Waveform Database, Version 3 Part 1 with the sampling rates of $250 \mathrm{~Hz}$. Each recording has 60 seconds of PPG signals. These subjects suffer with various types of heart abnormalities.

\subsection{Pre-processing}

For pre-processing, low pass filter which is Butterworth filter was used to filter out unwanted signals such as noise and baseline wandering. The Butterworth filter is a form of signal processing filter intended to have as a flat frequency response as possible in the pass band and also denoted to as a maximally flat magnitude filter. The choice of Butterworth as filter is because the advantage is it results in smooth, monotonically decreasing frequency response.

\subsection{Feature Extraction}

This stage is separated into two phases which are PPG segmentation and Cardioid based graph.

3.3.1. PPG Segmentation: The PPG signals are segmented by using MATLAB software. The purpose of segmentation is to identify the starting and end point of the waveform in order to plot Cardioid graph. Figure 4 shows one cycle of PPG signals. 


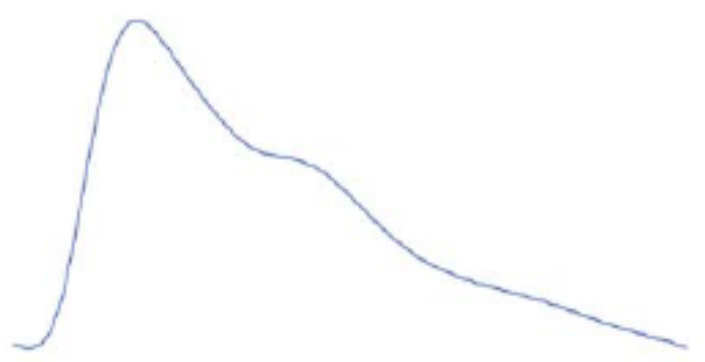

Figure 4. PPG Waveform [14]

3.3.2 Cardioid Based Graph: After attaining the PPG segments, the Cardioid based graph is created. This step is very significant in order to ensure the accurateness of the classification by using this technique. The PPG signal can be represented by $x(t)$ as in Equation 1.

$$
x(t)=\{x(1), x(2), x(3), \ldots, x(N)\}
$$

where, $x(t)=$ PPG waveforms and,

$N=$ the total number of PPG complexes for a given period.

The PPG complexes are then differentiated as in Equation 2 in order to obtain the points to form the Cardioid.

$$
y(t)=x(n)-x(n-1)
$$

where, $\mathrm{t}=1,2,3 \ldots(\mathrm{N}-1)$ and,

$y(t)=$ The differentiated PPG dataset.

A closed loop graph is then generated based on a scattered XY graph after obtaining the vectors of $\mathrm{x}$ and $\mathrm{y}$. The PPG amplitudes of the PPG signals are the $\mathrm{x}$-axis and the differentiated PPG values of $x$ are the $y$-axis. The time series representation is lost once the Cardioid has been generated and closed loop are formed as shown in Figure 5. and then the time series PPG signals is converted to a two dimensional loop and from the closed loop pattern, new features are extracted which are the centre coordinate of the graph called centroid and the distance of the centroid to a given point on the cardioid called extrema points.

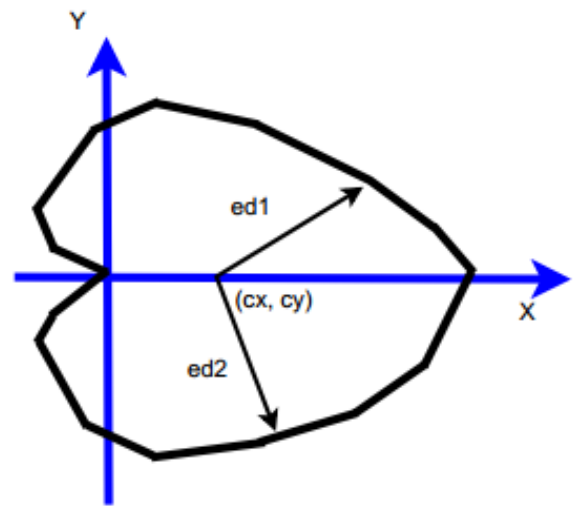

Figure 5. Cardioid Based Graph for Normal Heartbeat [15] 


\subsection{Classification}

For classification of the proposed system, a statistical approach is implemented. The results are evaluated by using two parameters which are area and standard deviation. The area of the Cardioid is obtained by applying the formula of a polygon. The standard deviation is then derived after calculating the area of the Cardioid. Both calculations are executed by using MATLAB. The purpose of this approach is to validate the accuracy of the propose technique by determining the differences between normal and abnormal heartbeats.

\section{Experimentation and Results}

In this section, the experimentation and result of the proposed detection technique is explained in more detail as shown in Figure 6. Referring to the Methodology section, the stages involved are Data Collection, Pre-processing, Feature Extraction and Classification stages.

Figure 6 shows the raw PPG signals of Subject 3 with heart abnormality as an example of the Data Collection stage.

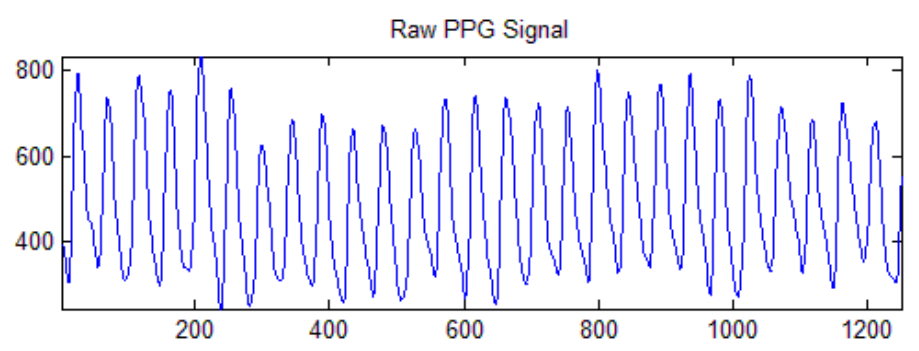

Figure 6. Raw PPG Signal of Subject 3 with Heart Abnormality

Figure 7 shows the filtered PPG signal using low pass filter. The objective of filtering is to remove the undesirable noise such as baseline wandering produced from the movement of the body. Besides that, it is also to enhance the preciseness of the quality of PPG signals. This step implements the second stage which is Pre-processing.

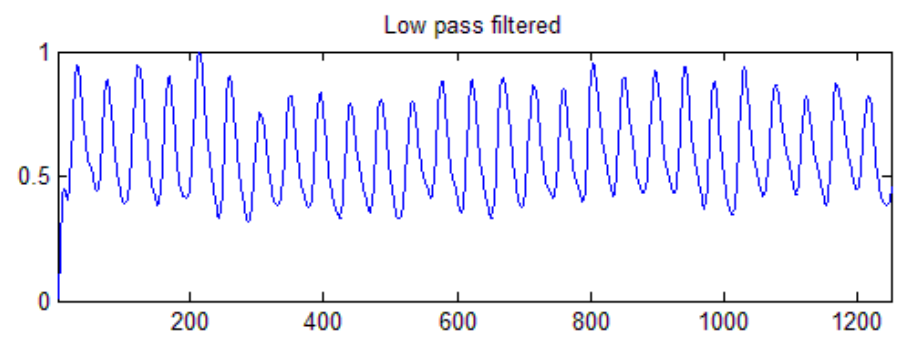

Figure 7. PPG Signal after Filtering using low Pass Filter

In order to segment the PPG after filtering process, Pan Tompkins algorithm was implemented as shown in Figure 8. This is the first part of the Feature Extraction stage whereas the second part involves the construction of the Cardioid graph. 


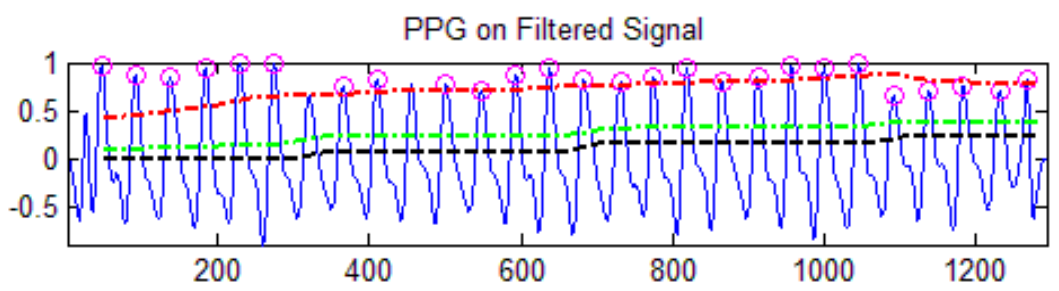

Figure 8. Segmentation of PPG Filtered Signal

A Cardioid graph is formed after the removal of the noise and segmentation as shown in Figure 9. In order to produce the Cardioid graph, two minimum points are identified, and their coordinates are taken.

From the figure, we can deduce that the shape of the graph is inconsistent with some smaller and bigger loops. This is due to the presence of normal and abnormal heartbeats. The aim of this step is to calculate the differences between normal and abnormal heartbeat. The looping of the graph for the normal PPG is consistent in shape as can be seen from Figure 9. The graph that is formed outside the constant shaped loop is the abnormal heart beat which shows the presence of heart abnormality.

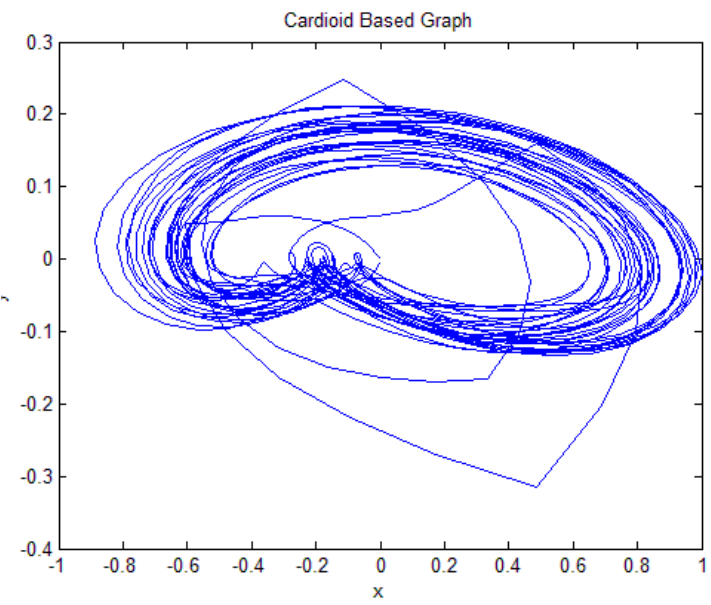

Figure 9. Cardioid Based Graph Subject 3 with Heart Abnormality

Two segmented PPG signal were taken for normal heartbeat and another two segmented PPG signal for abnormal heartbeat. Then, the Cardioid graph is formed to calculate its area and the standard deviation.

Figures 10, 11, 12 and 13 show the results from the segmented PPG signals of normal and abnormal heartbeat. From the figures we can see that, the area of the abnormal PPG signals is larger than the normal signals. The value of the standard deviation of normal PPG is also differs where the abnormal PPG signals where the standard deviation of abnormal PPG signal is higher than the normal PPG signal. The two values of standard deviation indicate which axis they originate where the right side is for the $\mathrm{x}$-axis and the left side is the $\mathrm{y}$-axis. 


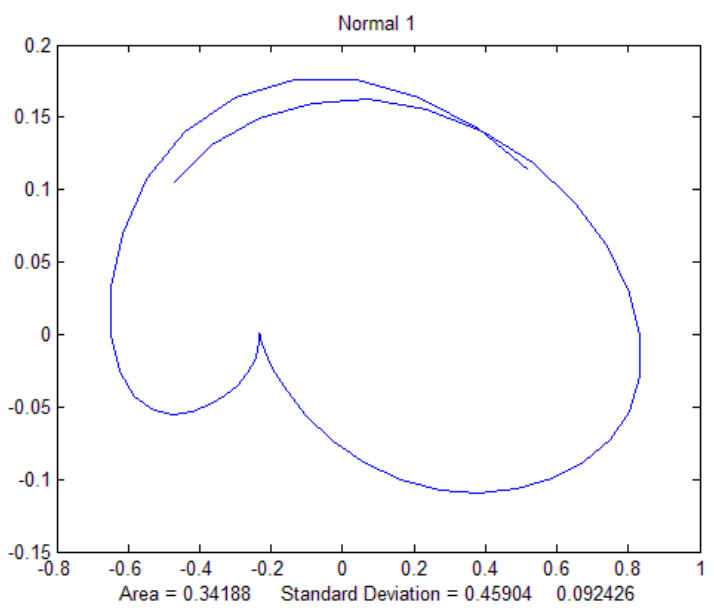

Figure 10. Result of the Segmented PPG Normal 1 Heartbeat

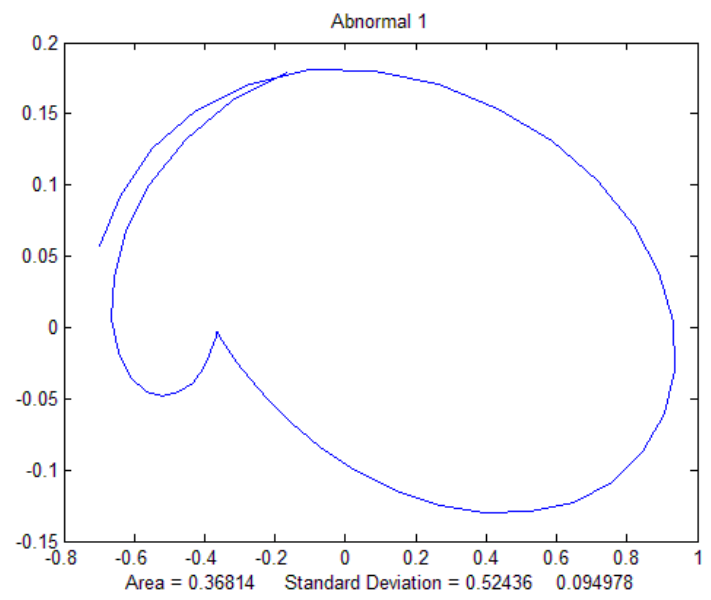

Figure 11. Result of the Segmented PPG Abnormal 1 Heartbeat

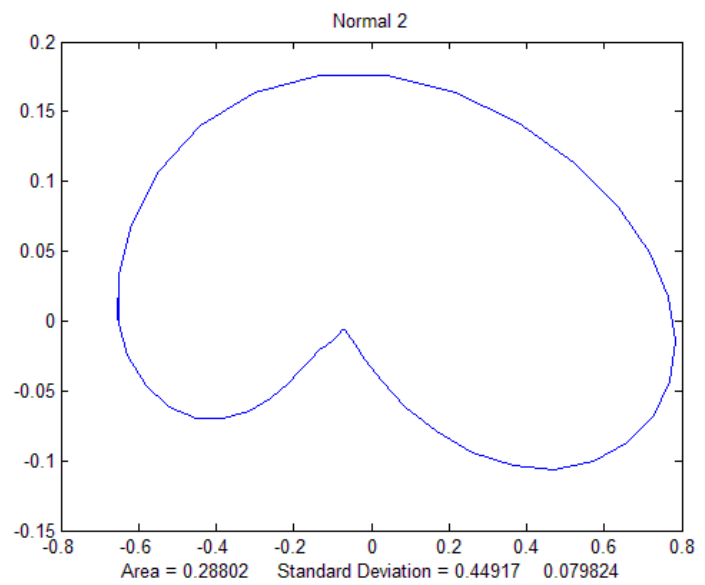

Figure 12. Result of the Segmented PPG Normal 2 Heartbeat 


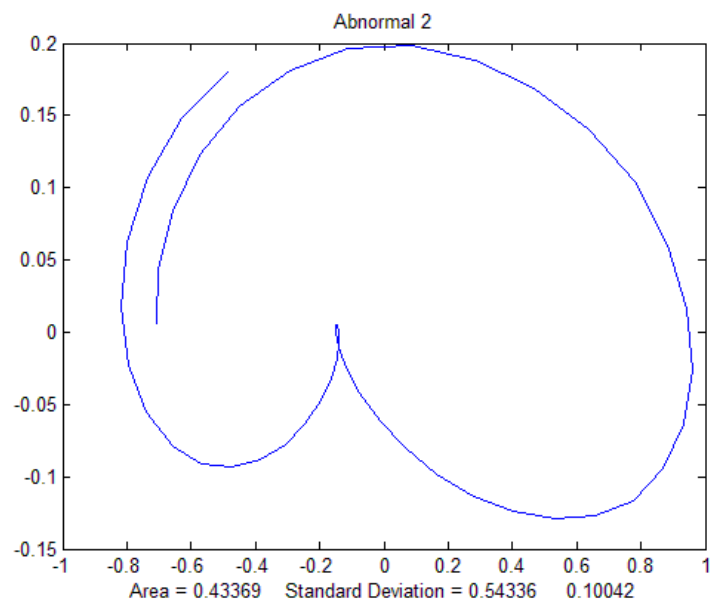

Figure 13. Result of the Segmented PPG Abnormal 2 Heartbeat

Table 1. Area of the Cardioid Based Graph

\begin{tabular}{|c|c|c|c|c|}
\hline Subject & $\begin{array}{c}\text { Normal } \\
\mathbf{1}\end{array}$ & $\begin{array}{c}\text { Normal } \\
\mathbf{2}\end{array}$ & $\begin{array}{c}\text { Abnormal } \\
\mathbf{1}\end{array}$ & $\begin{array}{c}\text { Abnormal } \\
\mathbf{2}\end{array}$ \\
\hline Subject 3 & 0.3419 & 0.2880 & 0.3681 & 0.4337 \\
\hline Subject 7 & 0.0951 & 0.1024 & 0.2394 & 0.2784 \\
\hline Subject 8 & 0.0503 & 0.0587 & 0.6122 & 0.1279 \\
\hline Subject 9 & 0.1590 & 0.1767 & 0.4022 & 0.4362 \\
\hline Subject 10 & 0.0667 & 0.0729 & 0.5636 & 0.1865 \\
\hline
\end{tabular}

Table 2. Standard Deviation of the Cardioid Based Graph

\begin{tabular}{|c|c|c|c|c|}
\hline Subject & Normal 1 & Normal 2 & Abnormal 1 & Abnormal 2 \\
\hline Subject 3 & $\mathrm{x}=0.4590$ & $\mathrm{x}=0.4492$ & $\mathrm{x}=0.5244$ & $\mathrm{x}=0.5434$ \\
$\mathrm{y}=0.0924$ & $\mathrm{y}=0.0798$ & $\mathrm{y}=0.0950$ & $\mathrm{y}=0.1004$ \\
\hline Subject 7 & $\mathrm{x}=0.2573$ & $\mathrm{x}=0.2538$ & $\mathrm{x}=0.4075$ & $\mathrm{x}=0.3964$ \\
$\mathrm{y}=0.0410$ & $\mathrm{y}=0.0413$ & $\mathrm{y}=0.0660$ & $\mathrm{y}=0.0693$ \\
\hline \multirow{2}{*}{ Subject 8 } & $\mathrm{x}=0.1628$ & $\mathrm{x}=0.1650$ & $\mathrm{x}=0.2799$ & $\mathrm{x}=0.2138$ \\
& $\mathrm{y}=0.1650$ & $\mathrm{y}=0.0261$ & $\mathrm{y}=0.0674$ & $\mathrm{y}=0.0372$ \\
\hline Subject 9 & $\mathrm{x}=0.2535$ & $\mathrm{x}=0.2655$ & $\mathrm{x}=0.3735$ & $\mathrm{x}=0.4026$ \\
& $\mathrm{y}=0.0413$ & $\mathrm{y}=0.0441$ & $\mathrm{y}=0.0639$ & $\mathrm{y}=0.0673$ \\
\hline \multirow{2}{*}{ Subject 10 } & $\mathrm{x}=0.1711$ & $\mathrm{x}=0.1715$ & $\mathrm{x}=0.3893$ & $\mathrm{x}=0.2913$ \\
& $\mathrm{y}=0.0284$ & $\mathrm{y}=0.0290$ & $\mathrm{y}=0.0882$ & $\mathrm{y}=0.0460$ \\
\hline
\end{tabular}

\section{Conclusion}

As a conclusion, we have established an effective and precise Cardioid based graph PPG heart abnormality detection technique. By using this approach, the area and standard deviation values of abnormal heartbeat gives twice the value than a normal heartbeat. All of these statistical parameters specify the dependability of the recommended method to detect the heart abnormality. Thus, this output indicates that Cardioid based graph PPG heart abnormality identification technique has the capability to discriminate between normal and abnormal heartbeat as an alternative to the current approaches. 


\section{References}

[1] Know The Fact About Heart Disease, http://www.cdc.gov/heartdisease/docs/consumered_heartdisease.pdf (Accessed on September 25th, 2015)

[2] Mozaffarian D, Benjamin EJ, Go AS, et al. Heart disease and stroke statistics-2015 update: a report from the American Heart Association. Circulation. 2015;131:e29-322.

[3] Test and Diagnosis of Heart Disease http://www.mayoclinic.org/diseases-conditions/heartdisease/basics/tests-diagnosis/con-20034056(Accessed on September 28th, 2015)

[4] Bolanos, M., H. Nazeran, and E. Haltiwanger. "Comparison of heart rate variability signal features derived from electrocardiography and photoplethysmography in healthy individuals." Engineering in Medicine and Biology Society, 2006. EMBS'06. 28th Annual International Conference of the IEEE. IEEE, 2006.

[5] Kumar, Mayank, Ashok Veeraraghavan, and Ashutosh Sabharval. "DistancePPG: Robust non-contact vital signs monitoring using a camera." Biomedical Optics Express 6.5 (2015): 1565-1588.

[6] Reisner, Andrew, et al. "Utility of the photoplethysmogram in circulatory monitoring." Anesthesiology 108.5 (2008): 950.

[7] Wijshoff, Ralph WCGR, et al. "Reducing motion artifacts in photoplethysmograms by using relative sensor motion: phantom study." Journal of biomedical optics 17.11 (2012): 117007-117007.

[8] Criado, Enrique, et al. "The role of air plethysmography in the diagnosis of chronic venous insufficiency." Journal of vascular surgery 27.4 (1998): 660-670.

[9] Peripheral Arterial Disease is Underdiagnosed in the Elderly, http://jmlevinemd.com/peripheralarterial-disease-is-underdiagnosed, (Accessed on 4th October 2015)

[10] Saad, Norhashimah Mohd, Abdul Rahim Abdullah, and Yin Fen Low. "Detection of heart blocks in ECG signals by spectrum and time-frequency analysis." Research and Development, 2006. SCOReD 2006. 4th Student Conference on. IEEE, 2006.

[11] Jose, S.K.; Shambharkar, C.M.; Chunkath, J., "Cardiac arrhythmia detection using ballistocardiogram signal," in Signal Processing, Informatics, Communication and Energy Systems (SPICES), 2015 IEEE International Conference on , vol., no., pp.1-5, 19-21 Feb. 2015

[12] Meza, M., "Preliminary study on detection of atrial fibrillation during oscillometric blood pressure measurement," in Information and Communication Technology, Electronics and Microelectronics (MIPRO), 2015 38th International Convention on , vol., no., pp.1174-1178, 25-29 May 2015

[13] Rubins, Uldis, et al. "Photoplethysmography analysis of artery properties in patients with cardiovascular diseases." 14th Nordic-Baltic Conference on Biomedical Engineering and Medical Physics. Springer Berlin Heidelberg, 2008.

[14] M. Elgendi. "On the Analysis of Fingertip Photoplethysmogram Signals", Current Cardiology Reviews, vol. 8, no. 1, pp. 14-25, 2012.

[15] Sidek, K. A., Jelinek, H.F.; Khalil, I., "Identification of Cardiac Autonomic Neuropathy patients using Cardioid based graph for ECG biometric," Computing in Cardiology, 2011, pp. 517-520, 18-21 Sept. 2011. 